Original article

\title{
Reasons of using complementary and alternative medicines (CAM) among elderly Malaysians of Kuala Lumpur and Selangor states: An exploratory study
}

\author{
Shahid Mitha ${ }^{\mathrm{a}}$, Vimalan Nagarajan ${ }^{\mathrm{b}}$, Muneer Gohar Babar ${ }^{\mathrm{a}}$, Mohammad Jamshed Ahmad Siddiqui ${ }^{\mathrm{c}}$, \\ Shazia Qasim Jamshed ${ }^{\mathrm{c}, *}$ \\ ${ }^{a}$ School of Dentistry, International Medical University, 57000 Kuala Lumpur, Malaysia \\ ${ }^{\mathrm{b}}$ Department of Pharmacy Practice, International Medical University, 57000 Kuala Lumpur, Malaysia \\ ${ }^{\mathrm{C}}$ Kulliyyah of Pharmacy, International Islamic University Malaysia, Kuantan Campus, Pahang 25200, Malaysia
}

\section{A R T I C L E I N F O}

\section{Article history:}

Received 31 March 2013

Accepted 28 May 2013

Available online 27 June 2013

\section{Keywords:}

Complementary and alternative medicine

Elderly

Belief

Attitude

Malaysian

\begin{abstract}
A B S T R A C T
Background: This study is aimed to explore the types of CAM and reasons of using CAM among elderly Malaysians.

Method: This cross-sectional study was conducted on a sample of 256 conveniently selected elderly Malaysians who were residing in the states of Selangor and Kuala Lumpur. A pre-validated interviewadministered questionnaire was used to gather information. Data was entered into PASW version 18 and analyzed.

Results: A total of 256 questionnaires were included in the study. A response rate of $64 \%$ was achieved. Out of 256 respondents, 92 (35.9\%) were male while 164 (64.1\%) were female. More than half of the respondents ( $n=141 ; 55.1 \%$ ) agreed that CAM is more effective than allopathic medicine. Chinese respondents showed strong belief in the effectiveness of CAM. In terms of safety of CAM, close to three quarters of respondents ( $n=178 ; 69.5 \%$ ) believed that CAM is safer than allopathic medicine. A large majority of respondents agreed that CAM has less side effects compared to allopathic medicine $(n=201$; $78.5 \%)$ and also agreed that CAM is good to maintain overall health and wellbeing ( $n=212 ; 82.8 \%$ ). A majority of the respondents expressed that they use CAM because allopathic medicine is less effective $(n=113 ; 44.1 \%)$

Conclusion: The current study reflects the reasons of using CAM among lay public from different ethnicities. There are no reports of adverse effects related to CAM use. Future approaches should be intended for awareness campaigns for consumers, highlighting safety profile of CAM and as well as forbidding their use without the consultation of healthcare professional.
\end{abstract}

Copyright $\odot$ 2013, InPharm Association, Published by Reed Elsevier India Pvt. Ltd. All rights reserved.

\section{Introduction}

Elderly is defined as those aged 60 years and above, as recommended by Malaysian Medical Association (MMA). According to the Department of Statistics'2000 Census, the Malaysian elderly population of 60 and above is increasing progressively and is predicted to increase to 3.2 million people by the year $2020 .{ }^{1}$ Although this indicates an increase in life expectancy, from healthcare perspective, this reflects that the population will be susceptible to different diseases and morbidities and, therefore, poses a higher demand for all medical and healthcare services. To be precise, elderly are always willing to search ways which improve their health and found to use

\footnotetext{
* Corresponding author.

E-mail addresses: pharmacist1992@live.com, jshazoo@gmail.com (S.Q. Jamshed).
}

complementary and alternative medicine (CAM) more often than other age groups. ${ }^{2,3}$ A study conducted in Australia showed that $60-80 \%$ of elderly consumers have declared use of at least one herbal or nutritional medicine on a regular basis. ${ }^{2}$

According to National Center for Complementary and Alternative Medicine (NCCAM), complementary and alternative medicine (CAM) is defined as a group of diverse medical and healthcare systems, practices, and products that are not generally considered part of conventional medicine. ${ }^{4}$ In Malaysia, CAM includes practices such as traditional Malay medicine, Islamic medical practice, traditional Chinese medicine, traditional Indian medicine, homeopathy, and complementary therapies, and excludes medical or dental practices utilized by registered medical or dental practitioners. ${ }^{5}$ The use of CAM in Malaysia is extensive ${ }^{6}$ and therefore maintained to be patronized by Malaysians in their efforts to seek treatment for diseases and as well as in maintaining health. ${ }^{5-9}$ In 
one Malaysian study it was reported that $69.4 \%$ of the Malaysian population used traditional and complementary medicines in their whole lifetime. ${ }^{8}$ The use of CAM in elderly is of particular interest because of several factors associated with old age such as polypharmacy, increased chronic diseases and decreased functional reserve. $^{2}$ Therefore, this study is aimed to explore the types of CAM and the reasons of using CAM among elderly Malaysians.

\section{Method}

\subsection{Study population, sampling, and sample size}

This is a cross-sectional study, which was conducted among the elderly population who are 60 years and above, residing in the states of Selangor and Wilayah Persekutuan (Kuala Lumpur). The survey was conducted from August 2012 to October 2012 among the lay public outside the shopping malls, post offices, schools, and banks.

Using Raosoft sample size calculator, the sample size was determined to be 377 with an $80 \%$ power and $95 \%$ confidence interval. The sample size was increased to 400 on account of a possible non-response rate. Therefore, 400 potential participants were conveniently contacted to participate in the study. Since the respondents were elderly, the method of interview-administered questionnaire was primarily used to facilitate respondents to answer questions as they might have difficulties in filling the questionnaire themselves. Their participation in the study was voluntary and signed informed consent was obtained from the respondents.

\subsection{Inclusion criteria}

Elderly people of more than sixty years of age, Malaysians who are residing in the states of Selangor and Kuala Lumpur and currently using or had previously used CAM in the past three months.

\subsection{Exclusion criteria}

Participants less than sixty years of age; residents from other states except Selangor and Kuala Lumpur; Non-CAM users, and healthcare practitioners.

\subsection{Research instrument}

The quantitative survey instrument was formed on the basis of available literature. ${ }^{9-11}$ The survey instrument was subjected to 8 faculty members working in Pharmacy and Chiropractic Department, International Medical University (IMU). This was then reviewed in the light of their feedback and thus revised. To ascertain the reliability of the instrument, a pilot study was conducted on 20 elderly Malaysians who were contacted outside the schools and hospitals on two different occasions with one week interval. After assessing the similarities and differences in response at each occasion, the validity of the instrument was ascertained. There was no significant difference observed during this time period. For the reliability of the questionnaire the pilot data was subjected to enter in PASW version 18 and Cronbach's alpha was computed. This was found to be 0.70 .

The questionnaire consisted of three parts. The first part, which consists of 15 questions, was about demographic and background characteristics of the participants with their past and current medical history (chronic diseases) and information about the types of CAM used. The second and third parts addressed the beliefs and attitudes toward CAM use and consist of eight questions for each part. Beliefs and attitudes domains have response categories based on "agree", "disagree" and "unsure".

\subsection{Statistical analysis}

All the data were entered into the Statistical Package for Social Sciences (PASW, version 18). Descriptive statistics were performed to evaluate the demographic characteristics of the respondents. Chi-square was used to determine association between demographic and beliefs and attitude toward CAM. A $p$ value of less than 0.05 was considered to be statistically significant.

\section{Results}

A total of 256 questionnaires were included in the study. A response rate of $64 \%$ was achieved. Out of 256 respondents, 92 (35.9\%) were male while 164 (64.1\%) respondents were female. The majority of the respondents $(n=151 ; 59 \%)$ were in the age range of 60-69. With regard to the ethnicity of the respondents, 147 (57.4\%) were Chinese, 94 (36.7\%) were Indians and 15 (5.9\%) were Malays.

In terms of education level, majority of the respondents ( $n=102 ; 39.8 \%$ ) were from primary education followed by secondary education ( $n=80: 31.3 \%)$. In total, 169 (66.0\%) respondents were reported to be suffering from a chronic disease. Interestingly, all the respondents from Malay and Indian ethnicity $(n=109$; 42.6\%) reported diabetes and hypertension as their chronic diseases. Only less than one quarter from Chinese ethnicity $(n=60$; $23.4 \%$ ) reported asthma and hypertension. The number of respondents reported not to suffer from any chronic disease were all male respondents, fall under the age category of 60-69 and stated to use CAM only for their health promotion and wellbeing.

The detailed demographic characteristics and practice information are shown in Table 1.

\subsection{Use of CAM among elderly}

The most commonly used CAM was reported to be Traditional Chinese medicine (TCM) (31\%) followed by supplements (18\%). The percentage of utilization of other forms of CAM is shown in Fig. 1.

Table 1

Demographic characteristics of the respondents.

\begin{tabular}{llc}
\hline Characteristics & & Frequency (\%) \\
\hline Age range & $60-69$ & $151(59)$ \\
& $70-79$ & $74(28.9)$ \\
Gender & $80-89$ & $25(9.8)$ \\
& 90 and above & $6(2.3)$ \\
Ethnicity & Male & $92(35.9)$ \\
& Female & $164(64.1)$ \\
Occupation & Malay & $15(5.9)$ \\
& Chinese & $147(57.4)$ \\
& Indian & $94(36.7)$ \\
& Retired & $55(21.5)$ \\
& Business & $36(14.1)$ \\
Education level & Self-employed & $20(7.8)$ \\
& Never employed & $64(25.0)$ \\
& Not reported & $81(31.6)$ \\
& Not educated & $43(16.8)$ \\
& Primary & $102(39.8)$ \\
& Secondary & $80(31.3)$ \\
Suffering from chronic disease & University & $8(3.1)$ \\
& Not reported & $23(9.0)$ \\
& Yes & $169(66.0)$ \\
& No & $87(34.0)$ \\
\hline
\end{tabular}




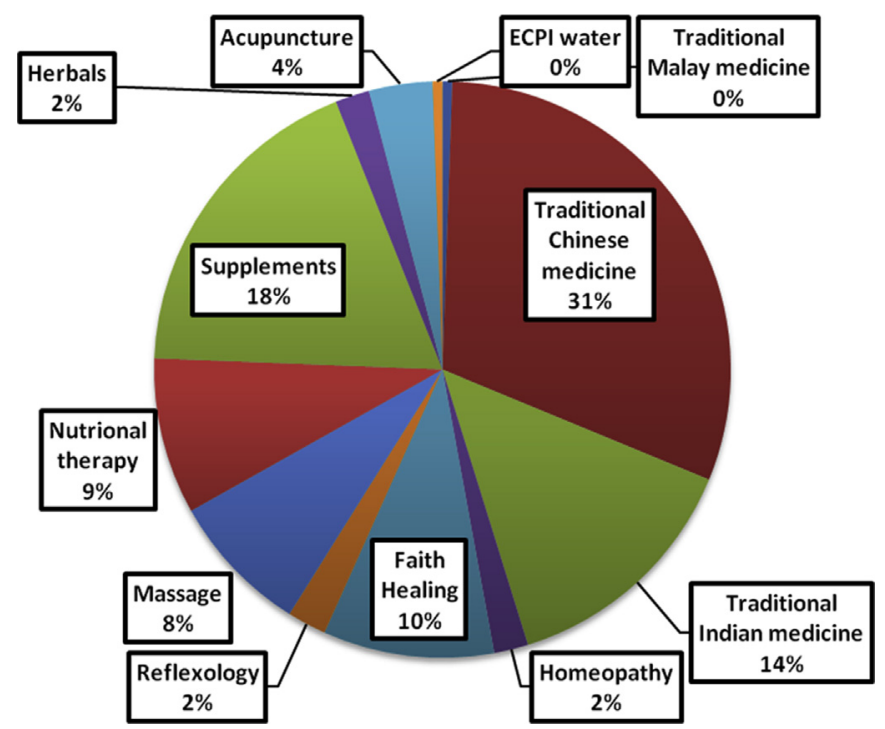

Fig. 1. Type of CAM ever used or currently using by respondents.

The majority of respondents used CAM for purpose of health promotion. A higher percentage of respondents consulted neither healthcare professional (81\%) nor CAM practitioner (78\%) before they use CAM. None of the respondents reported any side effects from CAM use.

\subsection{Beliefs of elderly toward CAM}

When the respondents were asked about the effectiveness of CAM, more than half of respondents $(n=141 ; 55.1 \%)$ agreed that CAM is more effective than allopathic medicine. This showed statistical significance with respect to ethnicity $(p=0.001)$. This belief is mainly held by Chinese respondents (76.7\%), although majority from Malays (66\%) and Indians (62.4\%) expressed the same. In terms of safety of CAM, close to three quarters of respondents ( $n=178 ; 69.5 \%$ ) believed that CAM is safer than allopathic medicine. This showed statistical significance with respect to gender $(p=0.006)$ and ethnicity $(p=0.001)$. Female respondents from Chinese ethnicity showed greater significant association. For detailed results please refer to Table 2 .

\subsection{Attitudes of elderly toward CAM}

Slightly less than half of the respondents expressed that they use CAM because allopathic medicine is less effective ( $n=113$; $44.1 \%)$. However, it was found almost half of the respondents declared that they use CAM because allopathic medicine is less safe
( $n=123 ; 48 \%$ ). This showed statistical significance with respect to age $(p=0.002)$ and ethnicity $(p=0.001)$. Elderly respondents in the age of 60-69 years and those from Chinese ethnicity expressed their stance that they use CAM because allopathic medicine is less safe. Majority of respondents denied that they use CAM because allopathic medicine is more expensive. Greater association was observed among Indian respondents. Further details of attitudes of elderly toward CAM use are shown in Table 3.

\section{Discussion}

The present study was designed to explore the reasons of using CAM among elderly Malaysians. The use of CAM by elderly is of particular interest in Malaysia due to the aging population and high prevalence of non-communicable diseases which requires lifelong treatment. It was found that approximately 66\% of CAM users were suffering from any kind of chronic disease. Presence of illness has been identified as a factor for CAM use in some studies. ${ }^{9,12-14}$ They might resort to CAM as a way to improve their health problems. ${ }^{13}$ It was found that females and ethnicity were indeed significant variables influencing CAM use. Similar findings were found in Singapore and Malaysia. ${ }^{14-17}$

TCM was identified as the one of the most commonly used CAM in the current study and this corroborates with the previous studies conducted in Singapore ${ }^{15}$ and Malaysia. ${ }^{9,18}$ The use was found to be fairly high in Malaysia presumably due to its easy accessibility. There are many TCM centers in both rural and urban Malaysia. This could be because Chinese had a strong faith in TCM. Besides that, Kuala Lumpur where the current research was executed primarily has significant Chinese population. Furthermore, in this study, the Chinese contributed to the highest percentage in terms of ethnic group.

A higher percentage of respondents never consulted any healthcare professional before CAM use. This could be due to lack of communication and also patient's attitude not to discuss CAM with their usual healthcare providers. ${ }^{9,17}$ Interestingly, none of the respondents in the current study have reported any side effects from CAM use. This in line with a Nigerian study where respondents believed herbal medicines possess no adverse effects, presumably due to ignorance. ${ }^{17}$

A higher percentage of respondents reported that CAM is safer than allopathic medication. These perceptions could be considered in the backdrop of general public opinion about herbal and traditional products being natural and safer to use. Interestingly a study conducted in Penang highlighted that traditional medicines are generally safe and natural. ${ }^{19}$

However, in terms of cost, the respondents in this study denied that they use CAM because allopathic medicine is more expensive. Many respondents from Indian ethnicity reported that CAM was at par, or more expensive than conventional medicines. Since there is

Table 2

Beliefs of elderly toward CAM use.

\begin{tabular}{|c|c|c|c|c|c|c|}
\hline Statements & Agree $n(\%)$ & Disagree $n(\%)$ & Unsure $n(\%)$ & Age $^{a}$ & Gender & $\mathrm{Eth}^{\mathrm{b}}$ \\
\hline I believe that CAM is more effective than allopathic medicine & $141(55.1)$ & $20(7.8)$ & $95(37.1)$ & 0.162 & 0.058 & $0.001^{*}$ \\
\hline I believe that CAM is safer than allopathic medicine & $178(69.5)$ & $8(3.1)$ & $70(27.3)$ & 0.065 & 0.006 & $0.001^{*}$ \\
\hline I believe that CAM is less expensive than allopathic medicine & $110(43.0)$ & $61(23.8)$ & $85(33.2)$ & 0.009 & 0.193 & $0.001^{*}$ \\
\hline I believe that CAM is a natural product & $213(83.2)$ & $2(0.8)$ & $41(16.0)$ & 0.172 & 0.052 & $0.015^{*}$ \\
\hline I believe that CAM has less side effects compared to allopathic medicine & $201(78.5)$ & $4(1.6)$ & $51(19.9)$ & 0.078 & 0.072 & $0.001^{*}$ \\
\hline I believe that CAM is good to maintain my overall health and wellbeing & $212(82.8)$ & $4(1.6)$ & $40(15.6)$ & 0.418 & 0.049 & $0.001^{*}$ \\
\hline I believe that CAM is better than allopathic medicine to maintain overall health & $164(64.1)$ & $4(1.6)$ & $88(34.4)$ & 0.167 & 0.086 & 0.051 \\
\hline I believe that CAM provides permanent cure for some disease & $96(37.5)$ & $19(7.4)$ & $141(55.1)$ & 0.063 & 0.430 & 0.127 \\
\hline
\end{tabular}

${ }^{*} p<0.05$.

a Age range: (60-69); (70-79); (80-89); 90 and above.

b Ethnicity: Malay, Chinese and Indian. 
Table 3

Attitudes of elderly toward CAM use.

\begin{tabular}{|c|c|c|c|c|c|c|}
\hline Statements & Agree $n(\%)$ & Disagree $n(\%)$ & Unsure $n(\%)$ & Age $^{a}$ & Gender & Eth $^{\mathrm{b}}$ \\
\hline I use CAM because allopathic medicine is less effective & $113(44.1)$ & $52(20.3)$ & $91(35.5)$ & 0.456 & 0.777 & $0.001^{*}$ \\
\hline I use CAM because allopathic medicine is less safe & $123(48.0)$ & $30(11.7)$ & $103(40.2)$ & 0.002 & 0.067 & $0.001^{*}$ \\
\hline I use CAM because allopathic medicine is more expensive & $73(28.5)$ & $75(29.3)$ & $108(42.2)$ & 0.158 & 0.089 & $0.001^{*}$ \\
\hline I use CAM because they are more natural compared to allopathic medicine & $199(77.7)$ & $0(0)$ & $57(22.3)$ & 0.013 & 0.041 & $0.001^{*}$ \\
\hline I use CAM because I have more faith in CAM & $160(62.5)$ & $7(2.7)$ & $89(34.8)$ & 0.120 & 0.872 & $0.001^{*}$ \\
\hline I use only CAM to maintain my health and wellbeing & $135(52.7)$ & $70(27.3)$ & $51(19.9)$ & 0.079 & 0.051 & $0.001^{*}$ \\
\hline I use CAM with allopathic medicine to maintain my health and wellbeing & $171(66.8)$ & $38(14.8)$ & $47(18.4)$ & 0.083 & 0.014 & $0.001^{*}$ \\
\hline I use CAM to restore the body's own balance & $203(79.3)$ & $4(1.6)$ & $49(19.1)$ & 0.067 & 0.020 & $0.001^{*}$ \\
\hline
\end{tabular}

${ }^{*} p<0.05$.

age range: (60-69); (70-79); (80-89); 90 and above.

b Ethnicity: Malay, Chinese and Indian.

a limited practice of Indian medicine such as Ayurvedic in Malaysia, the treatment cost might be higher compared to conventional medicines which could be obtained freely in public hospitals.

\section{Limitations of the study}

This study is conducted in two states of Malaysia and therefore cannot reflect the perceptions of elderly Malaysians in Eastern Peninsula and hence, generalizability is limited.

\section{Conclusion and recommendation}

The study reflects the reasons of using CAM among the elderly from different ethnicities. There are no reports of adverse effects related to CAM use. Future approaches should be intended for awareness campaigns for consumers, highlighting safety profile of CAM and as well as forbidding their use without consulting healthcare professional.

\section{Conflicts of interest}

All authors have none to declare.

\section{Acknowledgments}

We would like to acknowledge the research grant by International Medical University for funding this study.

\section{References}

1. Mafauzy M. The problems and challenging of the aging population of Malaysia. Malaysian J Med Sci. 2000;7:1-3.

2. Moses G. Complementary and alternative medicine use in the elderly. 34: 195200. J Pharm Pract Res. 2005;35:63-68.

3. McMahan S, Lutz R. Alternative therapy use among the young-old (ages 65 to 74): an evaluation of the MIDUS database. J Appl Gerontol. June 1, 2004;23. 91-103.
4. NCCAM. Complementary and Alternative Medicine: What People Aged 50 and Older Discuss with their Health Care Providers. AARP and NCCAM Survey Report. U.S. Department of Health and Human Services, National Institute of Health; 2011.

5. MOH. A Handbook of Traditional and Complementary Medicine Programme in Malaysia. Malaysia: Traditional and Complementary Medicine Division Ministry of Health; 2011.

6. Aziz Z, Tey NP. Herbal medicines: prevalence and predictors of use among Malaysian adults. Complement Ther Med. 2009;17:44-50.

7. Lee PY, Taha ABA, Lin K, Ghazali RS, Almashoor SHSA. Usage of complementary and alternative medicine among primary attendees, Kuching, Sarawah, Malaysia, January-April 2004. Asia Pac J Family Med. 2007;6.

8. Siti ZM, Tahir A, Farah AI, et al. Use of traditional and complementary medicine in Malaysia: a baseline study. 2009/12//. Complement Ther Med. 2009;17(5â€“6):292-299.

9. Huri HZ, Lian GTP, Hussain S, Pendek R, Widodo RT. A survey amongst complementary alternative medicine (CAM) users with type 2 diabetes. Int J Diab Metabol. 2009;17:15-19.

10. Lewith GT, Hyland ME, Shaw S. Do attitudes toward and beliefs about complementary medicine affect treatment outcomes?. 2013/05/19. Am J Public Health. 2002;92:1604-1606.

11. Onyiapat J-L, Okoronkwo I, Ogbonnaya N. Complementary and alternative medicine use among adults in Enugu, Nigeria. BMC Complement Altern Med. 2011;11:19.

12. Hasan SS, Ahmed SI, Bukhari NI, Loon WCW. Use of complementary and alternative medicine among patients with chronic diseases at outpatient clinics. Complement Ther Clin Pract. 2009;15:152-157.

13. Hassali MA, Saleem F, Shafie AA, et al. Assessment of general public perceptions toward traditional medicines used for aphrodisiac purpose in state of Penang, Malaysia. Complement Ther Clin Pract. 2012;18:257-260.

14. Alshagga MA, Al-Dubai SA, Faiq SSM, Yusuf AA. Use of complementary and alternative medicine among asthmatic patients in primary care clinics in Malaysia. Ann Thorac Med. 2011;6:115-119.

15. Lim MK, Sadarangani P, Chan HL, Heng JY. Complementary and alternative medicine use in multiracial Singapore. Complement Ther Med. 2005;13:16-24.

16. Saibul N, Shariff ZM, Rahmat A, Sulaiman S, Yaw YH. Use of complementary and alternative medicine among breast cancer survivors. Asian Pac J Cancer Prev. 2012;13:4081-4086.

17. Fakeye T, Adisa R, Musa I. Attitude and use of herbal medicines among pregnant women in Nigeria. BMC Complement Altern Med. 2009;9:53.

18. Hasan S, Yong C, Babar M, et al. Understanding, perceptions and self-use of complementary and alternative medicine (CAM) among Malaysian pharmacy students. BMC Complement Altern Med. 2011;11:95.

19. Babar ZU, Hassali MA, Lee ST, et al. An evaluation of consumers' perceptions regarding "modern medicines" in Penang, Malaysia. J Young Pharm. 2012;4: $108-113$ 